\title{
Predictors of calcification distribution in severe tricuspid aortic valve stenosis
}

\author{
Kerstin Piayda ${ }^{1}$. Lisa Dannenberg ${ }^{1}$ - Saif Zako ${ }^{1}$. Oliver Maier ${ }^{1}$. Georg Bosbach ${ }^{1}$. Amin Polzin ${ }^{1}$. Shazia Afzal ${ }^{1}$. \\ Christian Jung ${ }^{1} \cdot$ Ralf Westenfeld $^{1} \cdot$ Malte Kelm $^{1,2} \cdot$ Tobias Zeus $^{1} \cdot$ Verena Veulemans ${ }^{1}$ (i)
}

Received: 30 December 2020 / Accepted: 14 April 2021 / Published online: 20 April 2021

(c) The Author(s) 2021

\begin{abstract}
We investigated aortic valve calcification (AVC) distribution and predictors for leaflet calcification patterns in patients with severe tricuspid aortic valve stenosis undergoing transcatheter aortic valve replacement (TAVR). Patients undergoing routine multi-sliced computed tomography (MSCT) for procedural planning were enrolled. MSCT data were transferred to a dedicated workstation for evaluation (3mensio Structural Heart ${ }^{\mathrm{TM}}$, Pie Medical Imaging BV, Maastricht, The Netherlands) and analyzed. Participants were separated into asymmetrical (AC) and symmetrical (SC) leaflet calcification and potential predictors for calcification distribution were identified with univariate and multivariate regression analysis. 567 Participants with severe tricuspid AS were divided into asymmetrical (AC, $\mathrm{n}=443 ; 78.1 \%$ ) and symmetrical (SC, $\mathrm{n}=124 ; 21.9 \%)$ AVC. In AC, the non-coronary cusp was the most calcified cusp $(n=238 ; 57.7 \%)$. SC is more common in females (AC/SC: $49.2 \%$ vs. $67.7 \%$; $<0.0001$ ). AVC was more severe in patients with AC, who also have larger aortic root dimensions. Multivariate analysis depicted, inter alia, left ventricular outflow tract (LVOT) calcification $<25$ Agatston units (OR 1.81 [1.09-3.00], $\mathrm{p}=0.021$ ), a mean pressure gradient $<36 \mathrm{mmHg}$ (OR 1.77 [1.03-3.05], $\mathrm{p}=0.039)$, and an annulo-apical angle $>67^{\circ}(\mathrm{OR}$ 1.68 [1.00-2.80], $\mathrm{p}=0.049$ ) as predictors for $\mathrm{SC}$, although with only moderate predictive value. Data from this retrospective analysis indicate that $\mathrm{SC}$ occurs more frequently in females. The cumulative leaflet calcification burden is higher in patients with AC, who also present with larger aortic root dimensions. The predictive value for prominent calcification of different aortic valve cusps in AC patients was only low to moderate.
\end{abstract}

Trial registration number: NCT01805739.

Keywords Severe aortic stenosis $\cdot$ TAVR $\cdot$ Aortic valve calcification distribution $\cdot$ Multi-sliced computed tomography analysis · Leaflet calcification

\section{Introduction}

Aortic valve stenosis (AS) is the most common valvular heart disease in western countries [1]. The extent of aortic valve calcification (AVC), measured by multislice computed tomography (MSCT), correlates well with the degree of AS severity $[2,3]$, and is an integral part of current guideline recommendations for the management of patients with

Verena Veulemans

verena.veulemanns@med.uni-duesseldorf.de

1 Division of Cardiology, Pulmonology and Vascular Medicine, Medical Faculty, Heinrich Heine University, Moorenstr. 5, 40225 Düsseldorf, Germany

2 CARID (Cardiovascular Research Institute Düsseldorf), Moorenstr. 5, 40225 Düsseldorf, Germany valvular heart disease [4]. While sex- and severity-related distribution of aortic valve calcification are well-researched $[5,6]$, very little is known about predictors for (a)symmetrical leaflet calcification, which can be frequently observed in daily clinical practice.

Therefore, we performed an in-depth analysis of calcium distribution patterns and favoring, underlying conditions in patients with severe, tricuspid AS.

\section{Methods}

\section{Study population}

We retrospectively enrolled 567 patients with severe tricuspid AS, who underwent routine pre-procedural planning for 
transcatheter aortic valve replacement (TAVR) at the Heart Center Düsseldorf. Patients with prior aortic valve replacement and bicuspid aortic valve were excluded to guarantee comparability between groups. AS severity was defined according to the current European guideline recommendations for the management of patients with valvular heart disease [6]. Patients were separated into an asymmetrical (AC) and a symmetrical (SC) leaflet calcification pattern. In case of $\mathrm{AC}$, participants were further divided by the dominant calcified cusp (non-coronary; NCC, left coronary; LCC and right coronary cusp; RCC). Asymmetrical leaflet calcification was defined as a difference of $>150$ Agatston Units (AU) in-between the three leaflets, which is also visibly subsumable.

All patients provided written informed consent for the use of clinical, procedural, and follow-up data for research. The study procedures are in accordance with the Declaration of Helsinki. All necessary ethical oversight was secured: the Local Ethics Committee approved the study protocol (4080) and the study is registered at clinical trials (NCT01805739).

\section{Statistical analysis}

Continuous data are described by means with standard deviation, median with upper and lower 95\% confidence interval (CI) or interquartile ranges (IQR). Categorical variables are expressed by frequencies and percentages of a whole. Sexrelated differences are analysed with the 2-sided Student's t test for continuous variables and the chi-square test (Fisher's exact test) for categorical variables. Univariate and multivariate analysis are used to identify dependent and independent predictors for asymmetric and symmetric calcification distribution. Others calcification distribution patterns were excluded due to the small sample size (NCC/LCC; NCC/ RCC; LCC/RCC) Only co-variates with a p-value below 0.1 in the univariate analysis qualified for multivariate binary logistic regression. Receiver-operating-characteristic (ROC) curves are described as c-indices (area-under-the-curve) with $95 \%$ CI. The data analysis was performed with SPSS (version 22.0, SPSS, Inc., Chicago, IL) and GraphPad Prism (version 6.0, GraphPad Software, San Diego, CA). All statistical tests were 2 -sided, and a p-value $<0.05$ was considered statistically significant.

\section{MSCT imaging acquisition protocol and three- dimensional (3D) reconstruction}

Pre-procedural cardiac MSCT was routinely performed as native and contrast-enhanced multi-slice $\mathrm{CT}$ in all patients. CT data were obtained using a 128 -slice, single source CTscanner with temporal resolution of $150 \mathrm{~ms}$ and a collimation of $128 \times 0.6 \mathrm{~mm}$ ("SOMATOM Definition AS+", Siemens Healthcare, Forchheim, Germany). Images were taken in accordance with TAVR-related standardized recommendations for CT image acquisition [7]. They were transferred to a dedicated workstation for evaluation (3mensio Structural Heart ${ }^{\mathrm{TM}}$, Pie Medical Imaging BV, Maastricht, The Netherlands) and reconstructed in the coronal, sagittal and axial planes. After identification of the virtual annular plane, three hinge points were set on the axial plane, and 3D volume-rendered reconstruction initiated. We assessed the calcium amount of the aortic valve and the upper left ventricular outflow tract (LVOT) within $1 \mathrm{~cm}$ below the annulus. The calcification was re-calculated in Agatston Units (AU) for the overall- and separated leaflet calcium burden assessment. Calcium originating from extra-valvular structures, such as the mitral valve annulus, the ascending aorta, and the coronary arteries was cropped.

\section{Results}

\section{Baseline characteristics}

567 Patients were divided either into an asymmetrical (AC, $\mathrm{n}=443 ; 78.1 \%$ ) or a symmetrical (SC, $\mathrm{n}=124 ; 21.9 \%$ ) leaflet calcification pattern. In the AC group, the NCC was the most calcified cusp $(n=238 ; 57.7 \%)$, followed by the RCC $(n=72$; $12.7 \%)$ and the LCC $(n=58 ; 10.2 \%)$. Dominating NCC/ RCC calcification was observed in $6.3 \%(n=36)$ of patients, followed by a pronounced NCC/LCC calcification in $4.8 \%$ $(\mathrm{n}=27)$, and prominent $\mathrm{LCC} / \mathrm{RCC}$ calcification pattern in $2.1 \%(n=12)$ of cases.

SC was more common in females (AC/SC: $49.2 \%$ vs. $67.7 \% ; \mathrm{p}<0.0001)$ and was associated with various baseline characteristics such as immunosuppression (AC/SC: $2.7 \%$ vs. $8.7 \% ; \mathrm{p}=0.014)$ and chronic obstructive pulmonary disease (COPD) (AC/SC: $28.4 \%$ vs. $45.2 \%$; $\mathrm{p}=0.001$ ). Other comorbidities, rheological factors, and hemodynamic values were comparable between groups.

In patients with $\mathrm{AC}$, a lower wedge pressure $(\mathrm{AC} / \mathrm{SC}$ PCWP: $16.7 \pm 8.1 \mathrm{mmHg}$ vs. $20.5 \pm 5.1 \mathrm{mmHg}$; $\mathrm{p}=0.005$ ), a smaller aortic valve area (AC/SC AVA: $0.7 \pm 0.2 \mathrm{~cm}^{2}$ vs. $\left.0.8 \pm 0.2 \mathrm{~cm}^{2} ; \mathrm{p}=0.009\right)$, and higher transvalvular gradients (AC/SC mean pressure gradient: $62.2 \pm 23.2 \mathrm{mmHg}$ vs. $54.2 \pm 23.2 \mathrm{mmHg} ; \mathrm{p}=0.001$ ) were observed. Overall, patients with $\mathrm{AC}$ have an overall increased calcification burden $(\mathrm{AC} /$ SC: 2208 AU [1188-2906] vs. 1143 [495-1641]; p<0.001) an larger aortic root dimensions as compared to SC patients. Further baseline information are displayed in Table 1.

\section{Univariate and multivariate predictors for calcification distribution patterns}

\section{Symmetrical calcification}

Multivariate analysis depicted the presence of COPD (OR 2.15 [1.26-3.65], $\mathrm{p}=0.005)$, an LVOT calcification $<25 \mathrm{AU}$ 
Table 1 Patients' clinical and functional characteristics

Overall $(\mathrm{n}=567 ; 100 \%) \quad$ Asymmetrical (AC) $\quad$ Symmetrical (SC) $\quad$-value
$(\mathrm{n}=443 ; 78.1 \%) \quad(\mathrm{n}=124 ; 21.9 \%)$

Calcium distribution

Non-coronary cusp

Right coronary cusp

Left coronary cusp

Non-coronary cusp $=$ Right coronary cusp

Non-coronary cusp $=$ Left coronary cusp

Left coronary cusp $=$ Right coronary cusp

Clinical data

Age (years)

Female

Body mass index

Malignancy

Coronary artery disease

Previous percutaneous coronary intervention

Previous coronary artery bypass grafting

Arterial hypertension

Pulmonary hypertension

Diabetes mellitus

Insulin-dependent

Immunosuppression

Smoker

Previous pacemaker

Chronic obstructive pulmonary disease

Atrial fibrillation

Porcelain aorta

Medication

Oral anticoagulation

Statin

Rheology

Creatinine (mg/dl)

Glomerular filtration rate $(\mathrm{ml} / \mathrm{min})$

Hemoglobin (g/dl)

Functional data

Log EuroSCORE (\%)

Cardiac index $\left(1 / \mathrm{min} / \mathrm{m}^{2)}\right.$

Severely reduced $\operatorname{LVF}(<35 \%)$

Aortic regurgitation $\geq \mathrm{II}^{\circ}$

Mitral stenosis $\geq \mathrm{II}^{\circ}$

Mitral regurgitation $\geq \mathrm{II}^{\circ}$

Tricuspid regurgitation $\geq \mathrm{II}^{\circ}$

Systolic pulmonary artery pressure $(\mathrm{mmHg})$

PCWP (mmHg)

PVR (dynes $/ \mathrm{cm}^{5}$ )

SVR (dynes $/ \mathrm{cm}^{5}$ )

LVEDP (mmHg)

Aortic valve area $\left(\mathrm{cm}^{2)}\right.$

Maximum pressure gradient $(\mathrm{mmHg})$

Mean pressure gradient $(\mathrm{mmHg})$

Multi-sliced computed tomography data

$\begin{array}{ll}238(42.0) & 238(42.0) \\ 72(12.7) & 72(12.7) \\ 58(10.2) & 58(10.2) \\ 36(6.3) & 36(6.3) \\ 27(4.8) & 27(4.8) \\ 12(2.1) & 12(2.1)\end{array}$

$81.8 \pm 5.7$

$302(53.3)$

$26.7 \pm 4.7$

14 (2.5)

$408(72.2)$

225 (39.7)

64 (11.3)

517 (91.2)

$350(61.8)$

173 (30.5)

72 (12.7)

22 (3.9)

95 (16.8)

77 (13.6)

182 (32.1)

228 (40.2)

51 (9.0)

237 (41.9)

353 (62.4)

$1.3 \pm 0.9$

$55.5 \pm 20.0$

$12.3 \pm 5.0$

$25.2 \pm 14.5$

$2.3 \pm 0.6$

44 (7.8)

99 (17.5)

38 (6.8)

126 (22.2)

100 (17.7)

$43.1 \pm 15.0$

$17.6 \pm 9.3$

$225.5 \pm 205.4$

$1821 \pm 670.4$

$21.6 \pm 8.8$

$0.8 \pm 0.2$

$60.5 \pm 23.4$

$37.3 \pm 15.3$
$81.8+5.6$

218 (49.2)

$26.6 \pm 4.5$

10 (2.3)

323 (73.2)

183 (41.3)

53 (12.0)

404 (91.1)

268 (60.6)

128 (28.9)

50 (11.3)

12 (2.7)

79 (17.8)

57 (12.9)

126 (28.4)

180 (40.6)

$40(9.0)$

$182(41.2)$

280 (63.3)

$1.3 \pm 0.8$

$56.0 \pm 20.2$

$12.4 \pm 5.6$

$25.3 \pm 14.9$

$2.4 \pm 0.6$

33 (7.4)

76 (17.8)

29 (6.6)

93 (20.9)

76 (17.1)

$42.6 \pm 14.9$

$16.7 \pm 8.1$

$225.2 \pm 217.2$

$1798 \pm 687.7$

$21.6 \pm 8.3$

$0.7 \pm 0.2$

$62.2 \pm 23.2$

$38.3 \pm 15.2$
-
-
-
-
-

$81.7 \pm 5.8$

0.804

84 (67.8)

$27.1 \pm 5.3$

4 (3.2)

85 (68.5)

42 (33.9)

$11(8.9)$

113 (91.2)

82 (66.1)

45 (36.3)

22 (17.7)

$10(8.1)$

16 (12.9)

20 (16.1)

56 (45.2)

48 (38.7)

$11(8.9)$

$<0.0001 *$

0.262

0.519

0.740

0.063

0.422

1.000

0.296

0.158

0.067

0.014

0.222

0.374

$0.001 *$

0.756

1.000

55 (44.4)

0.538

73 (58.9)

0.402

$1.3 \pm 1.1$

0.424

$53.8 \pm 19.6$

0.274

$12.1 \pm 1.6$

0.532

$24.8 \pm 13.2$

0.735

$2.3 \pm 0.5$

0.160

$11(8.9)$

0.789

23 (18.7)

0.791

28 (22.6)

0.839

33 (26.6)

0.222

24 (19.3)

0.595

$44.8 \pm 15.0$

0.238

$20.5 \pm 12.1$

$0.005 *$

$226.5 \pm 159.4$

0.964

$1894 \pm 609.6$

0.281

$21.6 \pm 10.3$

0.988

$0.8 \pm 0.2$

$0.009 *$

$54.2 \pm 23.2 \quad 0.001 *$

$33.9 \pm 15.6 \quad 0.006^{*}$ 
Table 1 (continued)

\begin{tabular}{|c|c|c|c|c|}
\hline & Overall $(\mathrm{n}=567 ; 100 \%)$ & $\begin{array}{l}\text { Asymmetrical (AC) } \\
(\mathrm{n}=443 ; 78.1 \%)\end{array}$ & $\begin{array}{l}\text { Symmetrical (SC) } \\
(\mathrm{n}=124 ; 21.9 \%)\end{array}$ & p-value \\
\hline Aortic valve calcification (AU) & $1976[952-2658]$ & 2208 [1188-2906] & $1143[495-1641]$ & $<0.0001 *$ \\
\hline Non-coronary cusp (AU) & 829 [324-1175] & 964 [474-1283] & $352[139-466]$ & $<0.0001 *$ \\
\hline Left coronary cusp (AU) & $543[216-745]$ & $599[260-824]$ & $344[133-491]$ & $<0.0001 *$ \\
\hline Right coronary cusp (AU) & $583[227-763]$ & $655[270-843]$ & $326[115-432]$ & $<0.0001 *$ \\
\hline Left ventricular outflow tract (AU) & $188[0-186]$ & $182[0-188]$ & $209[1-120]$ & 0.481 \\
\hline Annulus area $\left(\mathrm{cm}^{2}\right)$ & $4.7 \pm 1.0$ & $4.8 \pm 1.0$ & $4.5 \pm 1.0$ & $0.001 *$ \\
\hline Perimeter $(\mathrm{mm})$ & $77.0 \pm 8.4$ & $77.6 \pm 8.6$ & $74.8 \pm 7.5$ & $0.001 *$ \\
\hline Annulus ellipticity & $1.2 \pm 0.1$ & $1.2 \pm 0.1$ & $1.2 \pm 0.1$ & 0.121 \\
\hline Left ventricular outflow tract mean (mm) & $23.6 \pm 2.8$ & $23.8 \pm 2.7$ & $22.9 \pm 2.8$ & $0.001 *$ \\
\hline Left ventricular outflow tract ellipticity & $1.4 \pm 0.2$ & $1.4 \pm 0.2$ & $1.4 \pm 0.2$ & $0.030 *$ \\
\hline Sinotubular junction mean (mm) & $29.1 \pm 3.3$ & $29.3 \pm 6.4$ & $28.3 \pm 3.2$ & 0.105 \\
\hline AOA mean $(\mathrm{mm})$ & $33.4 \pm 3.5$ & $33.4 \pm 3.5$ & $33.4 \pm 3.6$ & 0.886 \\
\hline Sinus of Valsalva diameter (mm) & $35.1 \pm 9.3$ & $35.3 \pm 9.3$ & $34.2 \pm 9.3$ & 0.259 \\
\hline Sinus of Valsalva radius NCC $(\mathrm{mm})$ & $17.9 \pm 5.0$ & $18.1 \pm 5.0$ & $18.4 \pm 4.9$ & 0.167 \\
\hline Sinus of Valsalva radius $\mathrm{LCC}(\mathrm{mm})$ & $17.8 \pm 4.9$ & $17.9 \pm 4.9$ & $17.4 \pm 4.7$ & 0.346 \\
\hline Sinus of Valsalva radius $\mathrm{RCC}(\mathrm{mm})$ & $17.1 \pm 4.7$ & $17.2 \pm 4.7$ & $16.6 \pm 4.7$ & 0.186 \\
\hline Right coronary artery distance $(\mathrm{mm})$ & $15.0 \pm 3.1$ & $15.1 \pm 3.1$ & $14.7 \pm 3.1$ & 0.197 \\
\hline Left coronary artery distance $(\mathrm{mm})$ & $12.3 \pm 2.7$ & $12.4 \pm 2.7$ & $11.8 \pm 2.6$ & $0.029 *$ \\
\hline Right coronary cusp length (mm) & $10.5 \pm 2.1$ & $10.6 \pm 2.1$ & $10.2 \pm 1.8$ & $0.037 *$ \\
\hline Left coronary cusp length (mm) & $11.0 \pm 2.0$ & $11.1 \pm 2.0$ & $10.5 \pm 2.0$ & $0.005^{*}$ \\
\hline Ratio Right coronary artery/Right coronary cusp & $1.5 \pm 0.4$ & $1.5 \pm 0.4$ & $1.5 \pm 0.3$ & 0.784 \\
\hline Ratio Left coronary artery/Left coronary cusp & $1.2 \pm 0.3$ & $1.1 \pm 0.3$ & $1.1 \pm 0.2$ & 0.812 \\
\hline Aortic root angulation $\left(^{\circ}\right)$ & $48.9 \pm 10.7$ & $49.3 \pm 10.3$ & $47.7 \pm 11.8$ & 0.191 \\
\hline Annulo-apical angulation $\left({ }^{\circ}\right)$ & $67.5 \pm 13.7$ & $67.1 \pm 13.2$ & $69.1 \pm 15.3$ & 0.208 \\
\hline
\end{tabular}

*Significant level $\mathrm{p}<0.05$ (bold, italics)

Values are mean $\pm \mathrm{SD}$, mean \pm 25 th and 75 th percentile or $\mathrm{n}(\%)$

$A U$ Agatston units, $B M I$ body mass index, $C A B G$ coronary artery bypass graft, $C A D$ coronary artery disease, $C I$ cardiac index, $C O P D$ chronic obstructive pulmonary disease, CVD cerebrovascular disease, dPmean/max mean/max. transvalvular gradient, LCCLeft coronary cusp, LVEDP Left ventricular enddiastolic pressure, $L V E F$ Left ventricular ejection fraction, $L A O$ left anterior oblique, $L V O T$ Left ventricular outflow tract, NCC Non-coronary cusp, PCI percutaneous coronary intervention, PHT pulmonary hypertension, RCCRight coronary cusp, SOVSinus of Valsalva, STJSinotubular junction

(OR 1.81 [1.09-3.00], $\mathrm{p}=0.021)$, a mean gradient below 36 $\mathrm{mmHg}$ (OR 1.77 [1.03-3.05], $\mathrm{p}=0.039)$, and an annulo-apical angulation above $67^{\circ}$ (OR 1.68 [1.00-2.80], $\mathrm{p}=0.049$ ) as predictive for a SC pattern. However, c-statistics-even when combined-only showed a moderate correlation (Table 2).

\section{Asymmetric calcification with dominating NCC calcification}

Univariate analysis depicted the presence of malignancy, male gender, the absence of a porcelain aorta, previous coronary artery bypass grafting, and a larger aortic anatomy as possible influencing factors for dominant NCC calcification. Multivariate analysis identified the absence of a porcelain aorta (OR 2.03 [1.07-3.86], $\mathrm{p}=0.031)$ and previous coronary artery bypass grafting (OR 1.95 [1.14-3.32], $\mathrm{p}=0.0014)$ as independent predictors for a pronounced NCC-calcification. However, c-statistics - even combinedremained only in a poor range (Table 2 ).

\section{Asymmetric calcification with dominating LCC calcification}

Prominent LCC-calcification was linked to a porcelain aorta, a smaller aortic valve area, higher pressure gradients and pronounced LVOT calcification. Multivariate analysis offered no independent predictor for dominating LCC-calcification in patients with AC pattern.

\section{Asymmetric calcification with dominating RCC calcification}

Concerning RCC calcification univariate analysis identified, inter alia, the absence of a permanent pacemaker (PPM) at 
Table 2 Discrimination performance (ROC and AUC statistics)

\begin{tabular}{|c|c|c|c|c|c|}
\hline Calcification site & Parameters & AUC & p-value & Lower $95 \%$ CI & Upper $95 \%$ CI \\
\hline \multirow[t]{5}{*}{ Symmetrical } & Chronic obstructive pulmonary disease & 0.58 & $0.025 *$ & 0.51 & 0.65 \\
\hline & Left ventricular outflow tract $<25 \mathrm{AU}$ & 0.60 & $0.005 *$ & 0.53 & 0.66 \\
\hline & Mean pressure gradient $<36 \mathrm{mmHg}$ & 0.59 & $0.009 *$ & 0.52 & 0.66 \\
\hline & Annulo-apical angulation $>67^{\circ}$ & 0.56 & 0.089 & 0.49 & 0.63 \\
\hline & Combined AUC & 0.68 & $<0.0001 *$ & 0.62 & 0.74 \\
\hline \multirow[t]{3}{*}{ Non-coronary cusp } & Previous coronary artery bypass grafting & 0.53 & 0.233 & 0.48 & 0.58 \\
\hline & No porcelain aorta & 0.53 & 0.342 & 0.48 & 0.58 \\
\hline & Combined AUC & 0.55 & $0.05 *$ & 0.50 & 0.60 \\
\hline Left coronary cusp & - & - & - & - & - \\
\hline \multirow[t]{5}{*}{$\mathrm{RCC}$} & No permanent pacemaker & 0.56 & 0.157 & 0.48 & 0.63 \\
\hline & Annulus ellipticity $<1.22$ & 0.60 & $0.012 *$ & 0.52 & 0.68 \\
\hline & $\begin{array}{l}\text { Ratio right coronary artery/right coronary } \\
\text { cusp }>1.43\end{array}$ & 0.58 & $0.040 *$ & 0.51 & 0.66 \\
\hline & Annulo-apical angulation $<67^{\circ}$ & 0.57 & 0.099 & 0.49 & 0.64 \\
\hline & Combined AUC & 0.68 & $<0.0001^{*}$ & 0.61 & 0.75 \\
\hline
\end{tabular}

*Significant level $\mathrm{p}<0.05$ (bold, italics)

Values are mean $\pm \mathrm{SD}$, mean \pm 25 th and 75 th percentile or $\mathrm{n}(\%)$

$A U$ Agatston units, $C A B G$ coronary artery bypass graft, COPD chronic obstructive pulmonary disease, LCCLeft coronary cusp, LVOTLeft ventricular outflow tract, NCC Non-coronary cusp, PMPacemaker, RCA Right coronary artery, RCC Right coronary cusp, SOVSinus of Valsalva, STJSinotubular junction

baseline, an annulus ellipticity index $<1.22$, larger dimension of the sinus of Valsalva, a greater RCA-to-RCC leaflet ratio $(\mathrm{RCA} / \mathrm{RCC}>1.43)$ and an annulo-apical angulation $<67^{\circ}$ as potential predictors.

In a multivariate analysis the absence of PPM at baseline (OR 6.01 [1.40-25.78], $\mathrm{p}=0.016$ ), an annulus ellipticity < 1.22 (OR 2.78 [1.55-4.97], $\mathrm{p}=0.001$ ), an RCA/RCC leaflet ratio $>1.43$ (OR 2.04 [1.15-3.65], $\mathrm{p}=0.016)$, and an annulo-apical angulation $<67^{\circ}$ (OR 1.98 [1.11-3.55], $\mathrm{p}=0.022$ ) proved to be independent predictive factors for prominent RCC calcification. c-Statistics remained only in a moderate range (Table 2). A graphical illustration of the read-out is given in Fig. 1. Detailed results of uni- und multivariate regression analysis can be found in the Supplement (Table 3).

\section{Discussion}

To our knowledge, this is the first study with a systematic and in-depth analysis of AVC distribution, which includes comorbidities, hemodynamic parameters, and anatomical pre-dispositions of patients with severe tricuspid AS undergoing TAVR.

Our retrospective study revealed that:
- A symmetrical calcification pattern is more frequently observed in females who have narrow aortic root dimensions.

- Cumulative leaflet calcification is higher in patients with asymmetrical leaflet calcification patterns.

- Independent predictors for prominent calcification of single aortic valve cusps vary widely and have only poor to moderate predictive value:

- Independent predictors for symmetrical leaflet calcification are COPD, a less pronounced LVOT calcification, lower mean pressure gradients, and a more horizontal aorta.

- Independent predictors for pronounced NCC calcification are previous $\mathrm{CABG}$ and the absence of a porcelain aorta.

- Independent predictors for a prominent RCC calcification are the absence of a PPM, a less elliptical annulus index, a higher RCA/RCC ratio, and a less horizontal aorta.

\section{Asymmetrical vs. symmetrical AVC distribution}

Koshkelashvili et al. retrospectively analyzed 318 non-contrast axial chest CT scans of subjects aged over 65 years in an all-comers cohort. They could also show that the LCC was frequently the most calcified cusp [8]. The study was not performed in patients solely presenting with AS but provided important information on race-related differences in early calcification patterns. In our study, race-differences 


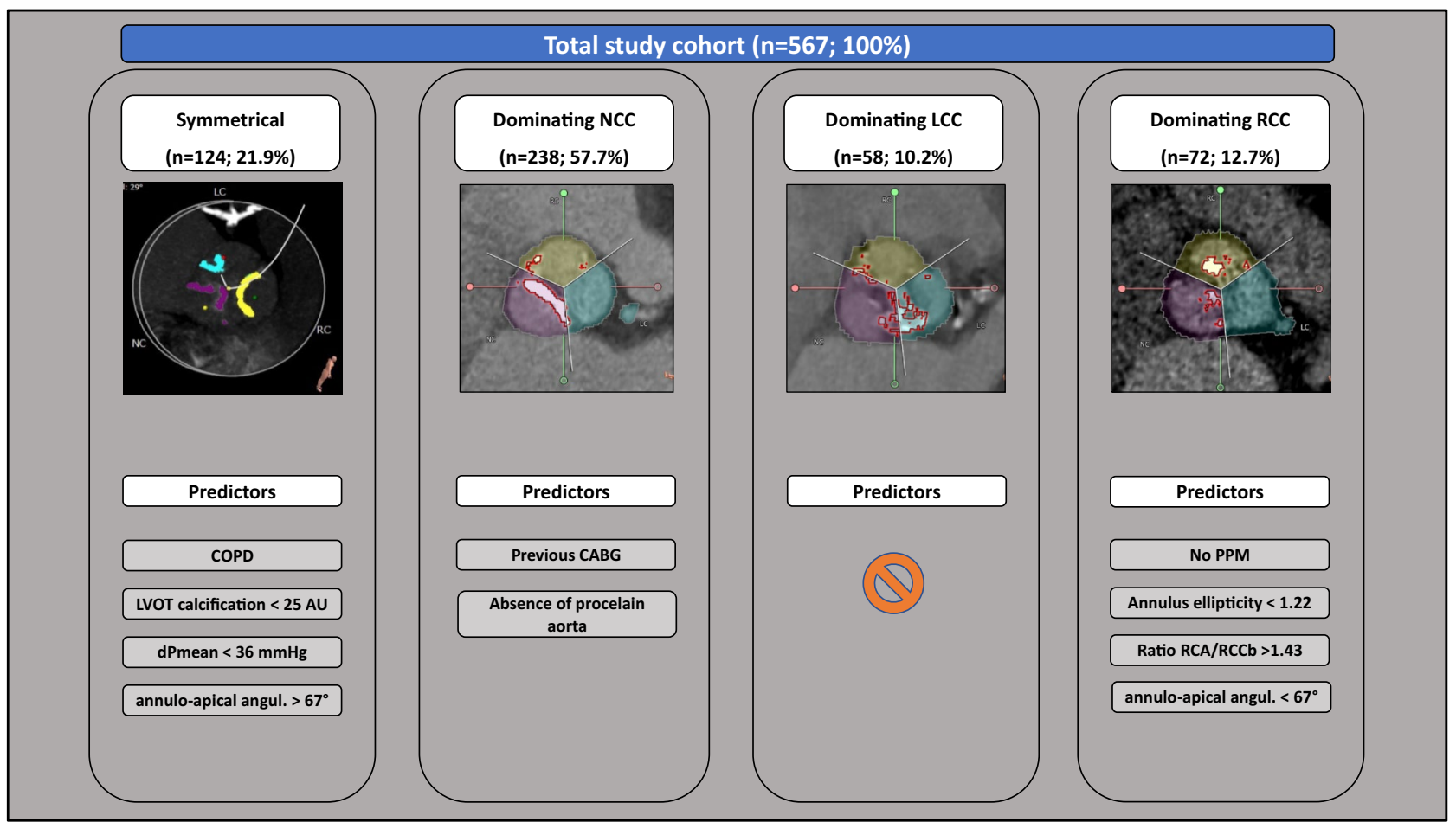

Fig. 1 Calcification distribution and independent predictors. The amount of calcium of the aortic valve and surrounding structures were assessed and re-calculated in Agatston Units (AU) for overalland separated leaflet calcium burden. Symmetrical and asymmetrical leaflet calcification with the dominant calcified cusp (non coronary cusp-magenta; left coronary cusp-blue; right coronary cusp-yellow) are displayed with independent predictors-identified by multivariate analysis. $C A B G$ coronary artery bypass grafting, $C O P D$ chronic obstructive pulmonary disease, dPmean mean pressure gradient, $P P M$ permanent pacemaker, Ratio $R C A / R C C b$ ratio of the right coronary artery to the base of the right coronary cusp

Woman have a slower progression rate of AS but lower calcium levels are already sufficient to create the same hemodynamic impairment as in men with higher AVC load [17]. This might be explained by the fact that women are more prone to higher levels of valvular fibrosis and dense connective tissue at the same degree of hemodynamic relevant aortic stenosis severity as compared to their male counterparts [18]. Since the SC group primarily consists of women, the lower aortic valve gradients and the lower overall AVC load may be explained by the aforementioned facts.

Shear forces, aortic root entrance angles, and flow-patterns may also play an important role in AVC distribution and should be further analyzed. A horizontal aorta, the extreme form of increased aortic root angulation and the annulo-apical entrance angles, is known to limit maneuverability of self-expandable devices $[19,20]$ but also means a potential shear force trigger, probably leading to flowdependent calcification. However, flow is pre-dominantly linked to myocardial function. Surprisingly, neither cardiac output, higher-grade reduced left ventricular ejection fraction or other hemodynamic characteristics in this context took a particular influence on calcification distribution, 
supposing that calcification may be more significantly linked to aortic flow characteristics.

\section{Dominant leaflet calcification in patients with AC}

Permanent ventricular pacing was shown to be associated with alterations in regional myocardial and coronary perfusion [21] and may, therefore, be linked to altered calcification patterns. Coronary artery disease was no dependent or independent predictor in our analysis, but the distribution of coronary artery disease and the distance of the coronary arteries might have an impact on AVC patterns through altered sinus perfusion and connected ostial calcification areas. Effacement of the sinuses may impair coronary flow and increase the mechanical stress and thus structural degeneration of the aortic valve leaflets [22]. In bicuspid valves, deterioration of aortic blood flow or eccentric flow is well-known to increase radial pressure and shear stress on the aortic wall [23], also causing enhanced dilatation of the aortic root, closing the circle towards aortic entrance angles and a horizontal aorta in tricuspid valves. A sub-analysis of the TAVI-WIN Registry [24] could show that increased calcium volumes of the RCC were an independent predictor for new pacemaker implantation after TAVR, whereas increased calcium volume of the NCC had a protective effect. However, findings are highly contradictive throughout current literature [14, 25]. Pathophysiologically, it remains unclear how different calcification patterns are created: in the initiation phase of disease, endothelial damage allows lipid infiltration and subsequent inflammation. In the propagation phase pro-fibrotic pathways and microcalcification smooth the way for further calcification [26]. Biomechanical studies could link high strain to the formation of calcific noduli and disease progression [27, 28]. Therefore, variable cusp geometries and congestive differences in length may also contribute to different calcification patterns, rather then underlying comorbidities.

\section{Limitations}

This is only a single-center analysis and limited to the retrospective quality of available data, which is also reflected by c-statistics. Furthermore, several important factors, like pressure recovery and ventriculo-arterial impedance, were not analyzed. This study of pre-disposing factors for different calcification patterns lacks translational value and does not influence clinical decision making.

\section{Future directions}

This is the first study trying to determine predictive factors for different types of aortic calcification patterns. Predictive factors vary widely throughout baseline characteristics and only showed poor to moderate correlation. This study may encourage clinicians to perform quantitative calcium assessment measurements in pre-TAVR MSCTs to create further evidence in this field.

\section{Conclusions}

Data from this retrospective analysis indicate that SC occurs more frequently in female patients and narrow aortic root anatomies, whereas cumulative leaflet calcification seems to be higher in $\mathrm{AC}$ patterns. Independent predictors for $\mathrm{SC}$ are COPD, a less pronounced LVOT calcification, lower mean pressure gradients and a more horizontal aorta. However, the correlation of different baseline characteristics with certain calcification patters were only in poor to moderate range. This is the first study addressing predictors for different calcification patterns, which are known to impact clinical outcomes of patients undergoing TAVR.

Supplementary Information The online version contains supplementary material available at https://doi.org/10.1007/s10554-021-02248-6.

Acknowledgements We thank Jenni Scharlau for substantial contribution in data acquisition.

Funding Open Access funding enabled and organized by Projekt DEAL.

Data availability Data will be made available up on reasonable request by the corresponding author.

\section{Declarations}

Conflict of interest Verena Veulemans, Tobias Zeus, Amin Polzin, and Ralf Westenfeld have received consulting fees, travel expenses, or study honoraries from Medtronic and Edwards Lifesciences. All other authors have nothing to disclose with regard to this project.

Ethical approval The study procedures are in accordance with the Declaration of Helsinki, and the Institutional Ethics Committee of the Heinrich-Heine University approved the study protocol (4080). The study is registered at clinical trials (NCT01805739).

Informed consent All patients provided written informed consent for use of clinical, procedural, and follow-up data for research and publication.

Open Access This article is licensed under a Creative Commons Attribution 4.0 International License, which permits use, sharing, adaptation, distribution and reproduction in any medium or format, as long as you give appropriate credit to the original author(s) and the source, 
provide a link to the Creative Commons licence, and indicate if changes were made. The images or other third party material in this article are included in the article's Creative Commons licence, unless indicated otherwise in a credit line to the material. If material is not included in the article's Creative Commons licence and your intended use is not permitted by statutory regulation or exceeds the permitted use, you will need to obtain permission directly from the copyright holder. To view a copy of this licence, visit http://creativecommons.org/licenses/by/4.0/.

\section{References}

1. Nkomo VT, Gardin JM, Skelton TN, Gottdiener JS, Scott CG, Enriquez-Sarano M (2006) Burden of valvular heart diseases: a population-based study. Lancet 368(9540):1005-1011

2. Messika-Zeitoun D, Aubry MC, Detaint D, Bielak LF, Peyser PA, Sheedy PF, Turner ST, Breen JF, Scott C, Tajik AJ, EnriquezSarano M (2004) Evaluation and clinical implications of aortic valve calcification measured by electron-beam computed tomography. Circulation 110:356-362

3. Cueff C, Serfaty JM, Cimadevilla C, Laissy JP, Himbert D, Tubach F, Duval X, Iung B, Enriquez-Sarano M, Vahanian A, MessikaZeitoun D (2011) Measurement of aortic valve calcification using multislice computed tomography: correlation with haemodynamic severity of aortic stenosis and clinical implication for patients with low ejection fraction. Heart 97:721-726

4. Baumgartner H, Falk V, Bax JJ, De Bonis M, Hamm C, Holm PJ, Iung B, Lancellotti P, Lansac E, Muñoz DR, Rosenhek R, Sjögren J, Mas PT, Vahanian A, Walther T, Wendler O, Windecker S, Zamorano JL (2017) 2017 ESC/EACTS Guidelines for the management of valvular heart disease. Eur Heart J 38(36):2739-2786

5. Clavel MA, Messika-Zeitoun D, Pibarot P, Aggarwal SR, Malouf J, Araoz PA, Michelena HI, Cueff C, Larose E, Capoulade R, Vahanian A, Enriquez-Sarano M (2013) The complex nature of discordant severe calcified aortic valve disease grading: new insights from combined Doppler echocardiographic and computed tomographic study. J Am Coll Cardiol 17(24):2329-2338

6. Aggarwal SR, Clavel MA, Messika-Zeitoun D, Cueff C, Malouf J, Araoz PA, Mankad R, Michelena H, Vahanian A, EnriquezSarano M (2013) Sex differences in aortic valve calcification measured by multidetector computed tomography in aortic stenosis. Circ Cardiovasc Imaging 6:40-47

7. Achenbach S, Delgado V, Hausleiter J, Schoenhagen P, Min JK, Leipsic JA (2012) SCCT expert consensus document on computed tomography imaging before transcatheter aortic valve implantation (TAVI)/transcatheter aortic valve replacement (TAVR). J Cardiovasc Comput Tomogr 6:366-380

8. Koshkelashvili N, Codolosa JN, Goykhman I, Romero-Corral A, Pressman GS (2015) Distribution of mitral annular and aortic valve calcium as assessed by unenhanced multidetector computed tomography. Am J Cardiol 15(12):1923-1927

9. Pawade T, Clavel MA, Tribouilloy C, Dreyfus J, Mathieu T, Tastet L, Renard C, Gun M, Jenkins WSA, Macron L, Sechrist JW, Lacomis JM, Nguyen V, Galian Gay L, Cuéllar Calabria H, Ntalas I, Cartlidge TRG, Prendergast B, Rajani R, Evangelista A, Cavalcante JL, Newby DE, Pibarot P, Messika Zeitoun D, Dweck MR (2018) Computed tomography aortic valve calcium scoring in patients with aortic stenosis. Circ Cardiovasc Imaging 11(3): 007146

10. Pawade T, Sheth T, Guzzetti E, Dweck MR, Clavel MA (2019) Why and how to measure aortic valve calcification in patients with aortic stenosis. JACC Cardiovasc Imaging 12(9):1835-1848. https://doi.org/10.1016/j.jcmg.2019.01.045
11. Erkapic D, De Rosa S, Kelava A, Lehmann R, Fichtlscherer S, Hohnloser SH (2012) Risk for permanent pacemaker after transcatheter aortic valve implantation: a comprehensive analysis of the literature. J Cardiovasc Electrophysiol 23:391-397

12. Binder RK, Webb JG, Toggweiler S, Freeman M, Barbanti M, Willson AB, Alhassan D, Hague CJ, Wood DA, Leipsic J (2013) Impact of post-implant SAPIEN XT geometry and position on conduction disturbances, hemodynamic performance, and paravalvular regurgitation. J Am Coll Cardiol Interv 6:462-468

13. Jilaihawi H, Makkar RR, Kashif M, Okuyama K, Chakravarty T, Shiota T, Friede G, Nakamura M, Doctor N, Rafique A, Shibayama K, Mihara H, Trento A, Cheng W, Friedman J, Berman D, Fontana GP (2014) A revised methodology for aortic-valvar complex calcium quantification for transcatheter aortic valve implantation. Eur Heart J Cardiovasc Imaging 15:1324-1332

14. Fujita B, Kütting M, Seiffert M, Scholtz S, Egron S, Prashovikj E, Börgermann J, Schäfer T, Scholtz W, Preuss R, Gummert J, Steinseifer U, Ensminger SM (2016) Calcium distribution patterns of the aortic valve as a risk factor for the need of permanent pacemaker implantation after transcatheter aortic valve implantation. Eur Heart J Cardiovasc Imaging 17:1385-1393

15. Maeno Y, Abramowitz Y, Kawamori H, Kazuno Y, Kubo S, Takahashi N, Mangat G, Okuyama K, Kashif M, Chakravarty T, Nakamura M, Cheng W, Friedman J, Berman D, Makkar RR, Jilaihawi H (2017) A highly predictive risk model for pacemaker implantation after TAVR. JACC Cardiovasc Imaging 10:1139-1147

16. Latsios G, Gerckens U, Buellesfeld L, Mueller R, John D, Yuecel S, Syring J, Sauren B, Grube E (2010) "Device landing zone" calcification, assessed by MSCT, as a predictive factor for pacemaker implantation after TAVI. Catheter Cardiovasc Interv 76:431-439

17. Nguyen V, Cimadevilla C, Estellat C, Codogno I, Huart V, Benessiano J, Duval X, Pibarot P, Clavel MA, Enriquez-Sarano M, Vahanian A, Messika-Zeitoun D (2015) Haemodynamic and anatomic progression of aortic stenosis. Heart 101:943-947

18. Simard L, Côté N, Dagenais F, Mathieu P, Couture C, Trahan S, Bossé Y, Mohammadi S, Pagé S, Joubert P, Clavel MA (2017) Sex-related discordance between aortic valve calcification and hemodynamic severity of aortic stenosis: is valvular fibrosis the explanation? Circ Res 120:681-691

19. Adams DH, Popma JJ, Reardon MJ (2014) Transcatheter aorticvalve replacement with a self-expanding prosthesis. N Engl J Med 370:1790-1798

20. Abramowitz Y, Maeno Y, Chakravarty T, Kazuno Y, Takahashi N, Kawamori H, Mangat G, Cheng W, Jilaihawi H, Makkar RR (2016) Aortic angulation attenuates procedural success following self-expandable but not balloon-expandable TAVR. JACC Cardiovasc Imaging 9:964-972

21. Skalidis EI, Kochiadakis GE, Koukouraki SI, Chrysostomakis SI, Igoumenidis NE, Karkavitsas NS, Vardas PE (2001) Myocardial perfusion in patients with permanent ventricular pacing and normal coronary arteries. J Am Coll Cardiol 37(1):124-129

22. Clavel MA, Pibarot P (2019) Functional and morphological interplay of the aortic valve, the aortic root, and the left ventricle. In: Surgical management of aortic pathology. Springer, Vienna

23. Bissell MM, Hess AT, Biasiolli L, Glaze SJ, Loudon M, Pitcher A, Davis A, Prendergast B, Markl M, Barker AJ, Neubauer S, Myerson SG (2013) Aortic dilation in bicuspid aortic valve disease: flow pattern is a major contributor and differs with valve fusion type. Circ Cardiovasc Imaging 6:499-507

24. Spaziano M, Chieffo A, Watanabe Y, Chandrasekhar J, Sartori S, Lefèvre T, Petronio AS, Presbitero P, Tchetche D, Iadanza A, Van Mieghem NM, Mehilli J, Mehran R, Morice MC, WIN-TAVI Investigators (2018) Computed tomography predictors of mortality, stroke and conduction disturbances in women undergoing TAVR: a sub-analysis of the WIN-TAVI Registry. J Cardiovasc Comput Tomogr 12:338-343 
25. Mauri V, Reimann A, Stern D, Scherner M, Kuhn E, Rudolph V, Rosenkranz S, Eghbalzadeh K, Friedrichs K, Wahlers T, Baldus S, Madershahian N, Rudolph TK (2016) Predictors of permanent pacemaker implantation after transcatheter aortic valve replacement with the SAPIEN 3. JACC Cardiovasc Interv 9(21):2200-2209

26. Peeters FECM, Meex SJR, Dweck MR, Aikawa E, Crijns HJGM, Schurgers LJ, Kietselaer BLJH (2018) Calcific aortic valve stenosis: hard disease in the heart: a biomolecular approach towards diagnosis and treatment. Eur Heart J 39:2618-2624

27. Halevi R, Hamdan A, Marom G, Lavon K, Ben-Zekry S, Raanani E, Haj-Ali R (2018) A new growth model for aortic valve calcification. J Biomech Eng 140(10):101008
28. Fisher CI, Chen J, Merryman WD (2013) Calcific nodule morphogenesis by heart valve interstitial cells is strain dependent. Biomech Model Mechanobiol 12:5-17

Publisher's note Springer Nature remains neutral with regard to jurisdictional claims in published maps and institutional affiliations. 